\title{
Effect of Magnetohydrodynamics and Couple Stress on Steady and Dynamic Characteristics of Plane Slider Bearing
}

\author{
Hanumagowda Bannihalli Naganagowda \\ School of Mathematics, REVA University \\ Rukmini Knowledge Park Bangalore - 560064 Karnataka - State India \\ *Corresponding author: hbn123@rediffmail.com
}

( Manuscript received 31 August 2015; accepted 25 January 2016; published 15 March 2016 )

\begin{abstract}
In this paper, an attempt has been made to study the effects of MHD and couple stress fluid lubricants on the steady state and dynamic behaviour of plane slider bearings by considering the squeezing action. The modified Reynolds equation is derived for the plane slider bearing with squeeze effects for the MHD and couple stress fluid. The dynamic stiffness and damping characteristics of one-dimensional MHD and couplestress plane slider bearings are computed. The effect of MHD and couple stress fluid lubricant improves the steady state and dynamic stiffness and damping characteristics of the plane slider bearings.
\end{abstract}

Keywords: squeeze film, magnetohydrodynamics, couple-stress fluid, steady and dynamic characteristics

\section{Introduction}

The MHD lubrication has been developed because the application of electric and magnetic fields increases the load carrying capacity of the liquid metal lubricated bearing. Last few years many types of magnetohydrodynamic (MHD) bearings have been investigated. The MHD lubrication in a slider bearing has been investigated by Agrawal [1] and found that for open-circuit case, load capacity increases due to inertia at small Hartmann number and decreases at large Hartmann number. Kuzma [2] presented the magnetohydrodynamic journal bearing. It is shown that the load carrying capacity is increased by the application of a magnetic field. Magneto-hydrodynamic couplestress squeeze film lubrication of circular stepped plates was studied by Naduvinamani et al., [3]. They observed that the effect of applied magnetic field on the squeeze film lubrication between circular stepped plates with couple stress fluids is to increase the load-carrying capacity significantly and to delay the time of approach considerably as compared to the corresponding nonmagnetic case. Dynamic characteristics for wide magneto-hydrodynamic slider bearings with a powerlaw film profile were presented by Lin [4]. He displayed that, comparing with the non-conducting-fluid powerlaw film-shape bearing, the MHD bearing provides an increase in the load capacity, and the stiffness and damping coefficients. Comparing with the MHD inclined-plane slider bearing, the MHD parabolic-film bearing signifies an improvement in the steady performances and the dynamic characteristics. Syeda Tasneem Fathima et al., [5] presented the analysis of Hydromagnetic squeeze films between porous rectangular plates with couple stress fluids. These authors predicted that effect of couplestresses on the MHD squeeze film lubrication between porous rectangular plates is to increase the load carrying capacity significantly and to delay the time of approach as compared to the corresponding non-magnetic case and Newtonian case.

Advances in technology and the severe operational requirements of machine necessitate the development of improved lubricants to ensure smooth operation. Generally the viscosity of the lubricating oils decreases with temperature. For operation under high speeds and heavy loads, oils containing high molecular weight polymers as viscosity index improvers are used to minimize viscosity variation with temperature. Conventionally, the prediction of squeeze film motion assumes that the lubricant behaves as a Newtonian viscous fluid. However, experimental results show that the addition of small amounts of long chained additives to a Newtonian fluid minimizes the sensitivity of the lubricant to change in shear rate and provides beneficial effects on the load-carrying and frictional characteristics [6-7]. Moreover, a base oil blended with additives can stabilize the behaviour of lubricants in elastohydrodynamic contacts and reduce friction and surface damage [8]. To describe the rheological 
behaviour of this kind of non-Newtonian lubricant, many microcontinum theories have been generated [9-10]. The Stokes theory [11] is the simplest generalization of the classical theory of fluids which allows for polar effects such as presence of couplestresses, body couples and non-symmetric tensors. This couplestress fluid is a special case of a non-Newtonian fluid and is intended to take account of particle size effects. On the basis of the microcontinum theory, this study is intended to predict the effects of couplestresses on the pure squeeze-film behaviour of a finite bearing subject to a time-dependent load. The modified Reynolds equation is obtained by using the Stokes equations to account for the influence of couplestresses resulting from the lubricant blended with various additives. Several types of couplestress bearings have been investigated [12-17].

Recently researchers Lin et al., [18] are analyzed the Dynamic stiffness and damping characteristics of one-dimensional magneto-hydrodynamic inclined-plane slider bearings. It will be shown that effect of applied magnetic field is to increase the bearing MHD steady load carrying capacity, the MHD dynamic stiffness and the MHD damping coefficient. In this paper, an attempt has been made to study the effects of MHD and couple stress fluid lubricants on the steady state and dynamic behaviour of plane slider bearings by considering the squeezing action and the results are compared with the MHD case studied by Lin et al. [18], and the results are presented in Table 1. The present results are in excellent agreement with the earlier studies Lin et al. [18]. To the best of the authors' knowledge, no studies are been found in the literatures on MHD steady and dynamic characteristics of plane slider bearings with couple stress fluids. This motivates the present study, where the main objective is to examine the effect of MHD and couple stress on steady and dynamic characteristics of plane slider bearings.

\section{Mathematical formulation and solution of the problem}

The geometry of the problem under consideration is shown in Fig. 1, in which lower surface have pure tangential sliding motion relative to the other surface. The film thickness in the entry region is $h_{1}$ and in the exit region is $h_{m}$. The two parallel plates of distance $h$ apart are approaching each other with a normal velocity $V(=$ $\partial h / \partial t)$. The uniform magnetic field $B_{0}$ is applied perpendicular to the two plates. The basic equations of motion for the steady laminar couple stress fluid flow in the film region in the presence of applied magnetic field are given by

$$
\begin{aligned}
& \mu \frac{\partial^{2} u}{\partial y^{2}}-\eta \frac{\partial^{4} u}{\partial y^{4}}-\sigma B_{0}^{2} u=\frac{\partial p}{\partial x}+\sigma E_{z} B_{0} \\
& \frac{\partial p}{\partial y}=0 \\
& \frac{\partial u}{\partial x}+\frac{\partial v}{\partial y}=0
\end{aligned}
$$

Table $1 \quad$ MHD and couple stress static and dynamic characteristics $W_{s}^{*}, K_{d}^{*}, D_{d}^{*}$ and comparison with the case studied by Lin et al., [18] with $\delta=1.5$

\begin{tabular}{cccccccc}
\hline & & & & \multicolumn{3}{c}{ Present analysis } \\
\cline { 5 - 7 } & \multicolumn{6}{c}{ Lin et al., [18] analysis } & \multicolumn{2}{c}{$l^{*}=0$} & $l^{*}=0.25$ \\
\hline & & & & \\
& $M_{0}$ & $h_{m}{ }^{*}=1$ & $h_{m}{ }^{*}=1.5$ & $h_{m}{ }^{*}=1$ & $h_{m}{ }^{*}=1.5$ & $h_{m}{ }^{*}=1$ & $h_{m}{ }^{*}=1.5$ \\
\hline & 0 & 0.158017 & 0.0706415 & 0.158003 & 0.0706352 & 0.169398 & 0.0734438 \\
$W_{s}{ }^{*}$ & 4 & 0.260152 & 0.136437 & 0.260153 & 0.136437 & 0.287065 & 0.147749 \\
& 8 & 0.425999 & 0.236413 & 0.426003 & 0.236415 & 0.507274 & 0.278785 \\
\hline & 0 & 0.295358 & 0.0988893 & 0.295332 & 0.0988806 & 0.332989 & 0.105628 \\
$K_{d}^{*}$ & 4 & 0.401149 & 0.148891 & 0.401151 & 0.148892 & 0.462482 & 0.164052 \\
& 8 & 0.593939 & 0.23806 & 0.593945 & 0.238062 & 0.724509 & 0.284157 \\
\hline & 0 & 0.210689 & 0.0941886 & 0.21067 & 0.0941803 & 0.225864 & 0.097925 \\
$D_{d}^{*}$ & 4 & 0.34687 & 0.181916 & 0.346871 & 0.181916 & 0.382754 & 0.196999 \\
& 8 & 0.567999 & 0.315217 & 0.568004 & 0.31522 & 0.676366 & 0.371714 \\
\hline
\end{tabular}




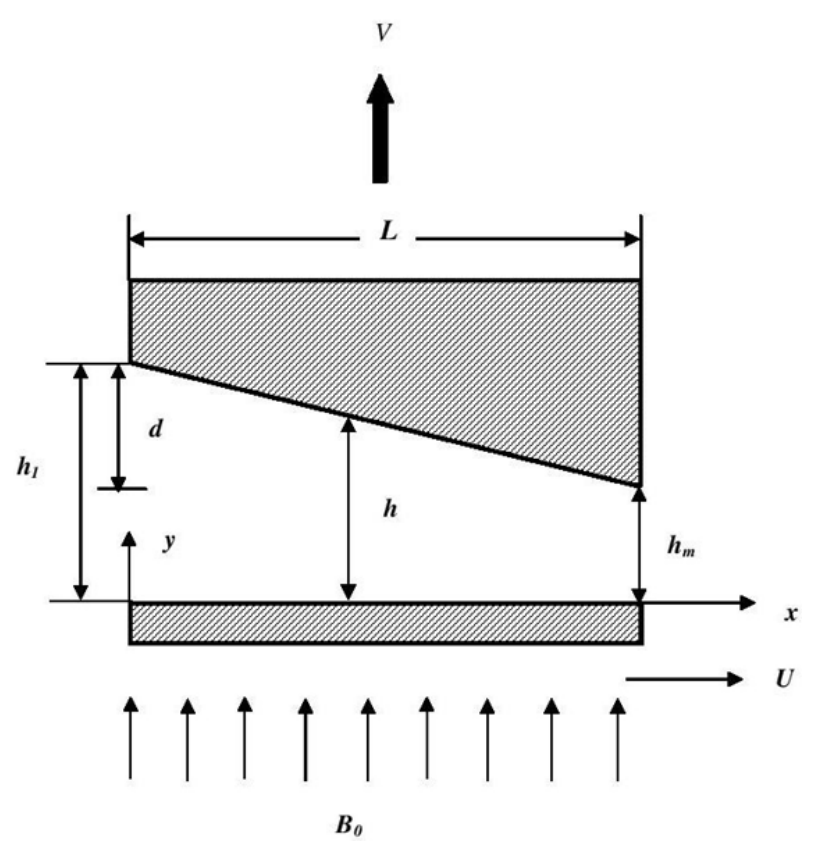

Fig. 1 Plane slider bearing

$$
\int_{y=0}^{h}\left(E_{z}+B_{0} u\right) \mathrm{d} y=0
$$

The film thickness for the inclined plane slider beating can be written as

$$
\begin{aligned}
& h(x, t)=h_{m}(t)+h_{s}(x) \\
& h_{s}(x)=d\left(1-\frac{x}{L}\right)
\end{aligned}
$$

where $h_{m}(t)$ is the minimum film thickness, $d$ is the difference of steady inlet-outlet thickness.
The relevant boundary conditions are

At the upper surface $y=h$

$$
u=0 \quad \frac{\partial^{2} u}{\partial y^{2}}=0 \quad v=\frac{\partial h}{\partial t}
$$

At the lower surface $y=0$

$$
u=U \quad \frac{\partial^{2} u}{\partial y^{2}}=0 \quad v=0
$$

Solving equation (1) using the boundary conditions (6), (7) and the condition (4), the velocity component is expressed as

$$
u=-\frac{U}{2} g_{1}-\frac{h_{m 0}^{2} h}{2 l \mu M_{0}^{2}} \frac{\partial p}{\partial x} g_{2}
$$

Where

$$
\begin{aligned}
& g_{1}=g_{11}-g_{12}, g_{2}=g_{13}-g_{14} \text { for } 4 M_{0}^{2} l^{2} / h_{m 0}^{2}<1 \\
& g_{1}=g_{21}-g_{22}, g_{2}=g_{23}-g_{24} \text { for } 4 M_{0}^{2} l^{2} / h_{m 0}^{2}=1 \\
& g_{1}=g_{31}-g_{32}, g_{2}=g_{33}-g_{34} \text { for } 4 M_{0}^{2} l^{2} / h_{m 0}^{2}>1
\end{aligned}
$$

Hartmann number $M_{0}=B_{0} h_{m 0}(\sigma / \mu)^{1 / 2}$ and Couple stress parameter $l=\sqrt{\eta / \mu}$

The associated relations in equations (9a), (9b) and (9c) are given in Appendix A.

Substituting for $u$ in to equation (3) and using the boundary conditions (6) and (7), the MHD and couplestress dynamic Reynolds equation is derived

$$
\frac{\partial}{\partial x}\left\{f\left(h, l, M_{0}\right) \frac{\partial p}{\partial x}\right\}=6 U \frac{\partial h}{\partial x}+12 \frac{\partial h}{\partial t}
$$

where

$$
f\left(h, l, M_{0}\right)= \begin{cases}\frac{6 h_{m 0}^{2} h^{2}}{\mu l M_{0}^{2}}\left\{\frac{A^{2}-B^{2}}{\left.\frac{A^{2}}{B} \tanh \frac{B h}{2 l}-\frac{B^{2}}{A} \tanh \frac{A h}{2 l}-\frac{2 l}{h}\right\}}\right. & \text { for } 4 M_{0}^{2} l^{2} / h_{m 0}^{2}<1 \\ \frac{6 h_{m 0}^{2} h^{2}}{\mu l M_{0}^{2}}\left\{\frac{2(\cosh (h / \sqrt{2} l)+1)}{3 \sqrt{2} \sinh (h / \sqrt{2} l)-h / l}-\frac{2 l}{h}\right\} & \text { for } 4 M_{0}^{2} l^{2} / h_{m 0}^{2}=1 \\ \frac{6 h_{m 0}^{2} h^{2}}{\mu l M_{0}^{2}}\left\{\frac{M_{0}\left(\cos B_{1} h+\cosh A_{1} h\right)}{h_{2}\left(A_{2} \sin B_{1} h+B_{2} \sinh A_{1} h\right)}-\frac{2 l}{h}\right\} & \text { for } 4 M_{0}^{2} l^{2} / h_{m 0}^{2}>1\end{cases}
$$

$A_{2}=\left(B_{1}-A_{1} \cot \theta\right), B_{2}=\left(A_{1}+B_{1} \cot \theta\right)$

Using the following non-dimensional quantities

$x^{*}=\frac{x}{L}, h^{*}\left(x^{*}\right)=\frac{h(x)}{h_{m 0}}, h_{s}^{*}\left(x^{*}\right)=\frac{h_{s}(x)}{h_{m 0}}$,

$\delta=\frac{\delta}{h_{m 0}}, P^{*}=\frac{p^{*} h_{m 0}^{2}}{\mu U L}$,

$l^{*}=\frac{2 l}{h_{m 0}}, t^{*}=\frac{t}{h_{m 0}}$ in equation (10), the non-dimensional MHD and couplestress Reynolds equation is expressed as 
$\frac{\partial}{\partial x^{*}}\left\{f\left(h^{*}, l^{*}, M_{0}\right) \frac{\partial P^{*}}{\partial x^{*}}\right\}=6 \frac{\mathrm{d}\left\{\delta\left(1-x^{*}\right)\right\}}{\mathrm{d} x^{*}}+12 \frac{\partial h^{*}}{\partial t^{*}}$

Where

$$
\begin{aligned}
& h^{*}\left(x^{*}, t^{*}\right)=h_{m}^{*}\left(t^{*}\right)+h_{s}^{*}\left(x^{*}\right) \\
& h_{s}^{*}\left(x^{*}\right)=\delta\left(1-x^{*}\right)
\end{aligned}
$$

$$
\begin{aligned}
& \left\{\frac{12 h^{* 2}}{l^{*} M_{0}^{2}}\left\{\frac{\left(A^{* 2}-B^{* 2}\right)}{\frac{A^{* 2}}{B^{*}} \tanh \frac{B^{*} h^{*}}{l^{*}}-\frac{B^{* 2}}{A^{*}} \tanh \frac{A^{*} h^{*}}{l^{*}}}-\frac{l^{*}}{h^{*}}\right\} \quad \text { for } M_{0}^{2} l^{*_{2}}<1\right. \\
& f^{*}\left(h^{*}, l^{*}, M_{0}\right)= \begin{cases}\frac{12 h^{* 2}}{l^{*} M_{0}^{2}}\left\{\frac{1+\cosh \left(\sqrt{2} h^{*} / l^{*}\right)}{(3 / \sqrt{2}) \sinh \left(\sqrt{2} h^{*} / l^{*}\right)-\left(h^{*} / l^{*}\right)}-\frac{l^{*}}{h^{*}}\right\} & \text { for } M_{0}^{2} l^{* 2}=1 \\
\frac{12 h^{* 2}}{l^{*} M_{0}^{2}}\left\{\frac{M_{0}\left(\cos B_{1}^{*} h^{*}+\cosh A_{1}^{*} h^{*}\right)}{A_{2}^{*} \sin B_{1}^{*} h^{*}+B_{2}^{*} \sinh A_{1}^{*} h^{*}}-\frac{l^{*}}{h^{*}}\right\} & \text { for } M_{0}^{2} l^{* 2}>1\end{cases} \\
& A^{*}=\left\{\frac{1+\left(1-l^{* 2} M_{0}^{2}\right)^{1 / 2}}{2}\right\}^{1 / 2}, B^{*}=\left\{\frac{1-\left(1-l^{* 2} M_{0}^{2}\right)^{1 / 2}}{2}\right\}^{1 / 2}
\end{aligned}
$$

$A_{1}^{*}=\sqrt{2 M_{0} / l^{*}} \cos \left(\theta^{*} / 2\right), \quad B_{1}^{*}=\sqrt{2 M_{0} / l^{*}} \sin \left(\theta^{*} / 2\right), \quad \theta^{*}=\tan ^{-1}\left(\sqrt{l^{* 2} M_{0}^{2}-1}\right)$

$A_{2}^{*}=\left(B_{1}^{*}-A_{1}^{*} \cot \theta^{*}\right), \quad B_{2}^{*}=\left(A_{1}^{*}+B_{1}^{*} \cot \theta^{*}\right)$

Integrating equation (12) w.r.t. $x^{*}$ twice, and using the pressure boundary conditions

$P^{*}=0$ at $x^{*}=0,1$ gives the MHD and couplestress film pressure

$$
P^{*}=6 G_{1}\left(0, x^{*}\right)+12 V^{*} G_{2}\left(0, x^{*}\right)-\left\{\frac{6 G_{1}(0,1)+12 V^{*} G_{2}(0,1)}{G_{3}(0,1)}\right\} G_{3}\left(0, x^{*}\right)
$$

where $V^{*}=\mathrm{d} h_{m}^{*} / \mathrm{d} t^{*}$ is the non-dimensional squeezing velocity, and

$$
\begin{aligned}
& G_{1}\left(0, x^{*}\right)=\int_{x^{*}=0}^{x^{*}} \frac{\delta\left(1-x^{*}\right)}{f^{*}\left(h^{*}, l^{*}, M_{0}\right)} \mathrm{d} x^{*}, G_{1}(0,1)=\int_{x^{*}=0}^{1} \frac{\delta\left(1-x^{*}\right)}{f^{*}\left(h^{*}, l^{*}, M_{0}\right)} \mathrm{d} x^{*} \\
& G_{2}\left(0, x^{*}\right)=\int_{x^{*}=0}^{x^{*}} \frac{x^{*}}{f^{*}\left(h^{*}, l^{*}, M_{0}\right)} \mathrm{d} x^{*}, G_{2}(0,1)=\int_{x^{*}=0}^{1} \frac{x^{*}}{f^{*}\left(h^{*}, l^{*}, M_{0}\right)} \mathrm{d} x^{*} \\
& G_{3}\left(0, x^{*}\right)=\int_{x^{*}=0}^{x} \frac{1}{f^{*}\left(h^{*}, l^{*}, M_{0}\right)} \mathrm{d} x^{*}, G_{3}(0,1)=\int_{x^{*}=0}^{1} \frac{1}{f^{*}\left(h^{*}, l^{*}, M_{0}\right)} \mathrm{d} x^{*}
\end{aligned}
$$

The load per unit width is given by $w=b \int_{0}^{L} p \mathrm{~d} x$

The non dimensional load carrying capacity is given by

$$
\begin{aligned}
& W^{*}=\frac{w h_{m 0}^{2}}{\mu U L^{2} b},=\int_{x^{*}=0}^{1} P^{*} \mathrm{~d} x^{*} \\
& W^{*}=6 \int_{0}^{1} \int_{x^{*}=0}^{x^{*}} \frac{\delta\left(1-x^{*}\right)}{f^{*}\left(h^{*}, l^{*}, M_{0}\right)} \mathrm{d} x^{*} \mathrm{~d} x^{*}+12 V^{*} \int_{0}^{1} \int_{x^{*}=0}^{x^{*}} \frac{x^{*}\left(h^{*}, l^{*}, M_{0}\right)}{f^{*} x^{*} \mathrm{~d} x^{*}} \\
& \quad-\left\{\frac{6 G_{1}(0,1)+12 V^{*} G_{2}(0,1)}{G_{3}(0,1)}\right\} \int_{0}^{1} \int_{x^{*}=0}^{x^{*}} \frac{1}{f^{*}\left(h^{*}, l^{*}, M_{0}\right)} \mathrm{d} x^{*} \mathrm{~d} x^{*}
\end{aligned}
$$


By letting the minimum film height be constant and letting squeezing velocity be zero in equations (15) and (16), the dimensionless steady film pressure $P_{s}^{*}$ and the dimensionless steady load carrying capacity $W_{s}^{*}$ is given by

$$
\begin{aligned}
& P_{s}^{*}=6 G_{1}\left(0, x^{*}\right)-6\left\{\frac{G_{1}(0,1)}{G_{3}(0,1)}\right\} G_{3}\left(0, x^{*}\right) \\
& W_{s}^{*}=6 \int_{0}^{1} \int_{x^{*}=0}^{x^{*}} \frac{\delta\left(1-x^{*}\right)}{f^{*}\left(h^{*}, l^{*}, M_{0}\right)} \mathrm{d} x^{*} \mathrm{~d} x^{*}-6\left\{\frac{G_{1}(0,1)}{G_{3}(0,1)}\right\} \int_{0}^{1} \int_{x^{*}=0}^{x^{*}} \frac{1}{f^{*}\left(h^{*}, l^{*}, M_{0}\right)} \mathrm{d} x^{*} \mathrm{~d} x^{*}
\end{aligned}
$$

Differentiating MHD film force partially with respect to dimensionless minimum film thickness and thereafter taking the value under steady state gives the dimensionless linear dynamic stiffness $\left(K_{d}^{*}\right)$ is given by

$$
\begin{aligned}
& K_{d}^{*}=-\left(\frac{\partial W_{s}^{*}}{\partial h_{m}^{*}}\right) \\
& =-6 \int_{x^{*}=0}^{1} \int_{x^{*}=0}^{x^{*}} h_{s}^{*}(-1) f^{*-2} \frac{\partial f^{*}\left(h^{*}, l^{*}, M_{0}\right)}{\partial h_{m}^{*}} \mathrm{~d} x^{*} \mathrm{~d} x^{*} \\
& +6 G \int_{x^{*}=0}^{1} \int_{x^{*}=0}^{x^{*}} \frac{1}{f^{*}\left(h^{*}, l^{*}, M_{0}\right)} \mathrm{d} x^{*} \mathrm{~d} x^{*} \\
& +6\left\{\frac{G_{1}(0,1)}{G_{3}(0,1)}\right\}_{x^{*}=0}^{1} \int_{x^{*}=0}^{x^{*}}(-1) f^{*-2} \frac{\partial f^{*}\left(h^{*}, l^{*}, M_{0}\right)}{\partial h_{m}^{*}} x^{*} \mathrm{~d} x^{*} \\
& G_{3}(0,1)\left(\int_{x^{*}=0}^{1} h_{s}^{*}(-1) f^{*-2} \frac{\partial f^{*}\left(h^{*}, l^{*}, M_{0}\right)}{\partial h_{m}^{*}} \mathrm{~d} x^{*}\right)-G_{1}(0,1)\left(\int_{x^{*}=0}^{1}(-1) f^{*-2} \frac{\partial f^{*}\left(h^{*}, l^{*}, M_{0}\right)}{\partial h_{m}^{*}} \mathrm{~d} x^{*}\right)
\end{aligned}
$$

where

$$
\begin{aligned}
& \frac{\partial f^{*}\left(h^{*}, l^{*}, M_{0}\right)}{\partial h_{m}^{*}}=\frac{12}{l^{*} M_{0}^{2}}\left\{\left(\frac{2 h^{*} \xi_{1}-h^{* 2} \xi_{2}}{\xi_{1}^{2}}\right)-l^{*}\right\} \\
& \xi_{1}=\xi_{11}, \quad \xi_{2}=\xi_{12} \text { for } M_{0}^{2} l^{*_{2}}<1 \\
& \xi_{1}=\xi_{21}, \quad \xi_{2}=\xi_{22} \text { for } M_{0}^{2} l^{*_{2}}=1 \\
& \xi_{1}=\xi_{31}, \quad \xi_{2}=\xi_{32} \text { for } M_{0}^{2} l^{* 2}>1
\end{aligned}
$$

The associated relations in equations (21a), (21b) and (21c) are given in Appendix A.

Differentiating MHD film force partially with respect to dimensionless squeezing velocity and thereafter taking the value under steady state gives the dimensionless linear damping coefficient $\left(D_{d}^{*}\right)$ is given by

$$
\begin{aligned}
D_{d}^{*} & =-\left(\frac{\partial W^{*}}{\partial V^{*}}\right) \\
& =-12 \int_{x^{*}=0}^{1} \int_{x^{*}=0}^{x^{*}} \frac{x^{*}}{f^{*}\left(h^{*}, l^{*}, M_{0}\right)} \mathrm{d} x^{*} \mathrm{~d} x^{*}+\frac{12 G_{2}(0,1)}{G_{3}(0,1)} \int_{x^{*}=0}^{1} \int_{x^{*}=0}^{x^{*}} \frac{1}{f^{*}\left(h^{*}, l^{*}, M_{0}\right)} \mathrm{d} x^{*} \mathrm{~d} x^{*}
\end{aligned}
$$

\section{Results and discussion}

The dynamic characteristics of plane slider bearings lubricated with an electrically conducting couple stress fluid with transverse magnetic field are analysed. Both the steady state performance and the dynamic characteristics are analysed. The present results are compared with one dimensional magneto-hydrodynamic inclined slider bearings studied by Lin et al. [18]. The dynamic characteristics of plane slider bearings lubricated with an electrically conducting couple stress fluid with transverse magnetic field are analysed with respect to the following various non-dimensional parameters: to 1.0

$$
M_{0}=0,4,8, l^{*}=0.0 .25,0.5, \delta=0.1 \text { to } 3.5, h_{m}{ }^{*}=0.4
$$

\subsection{Steady film pressure}

Figure 2 depicts the variation of non-dimensional steady film pressure, $P_{s}{ }^{*}$ with $x^{*}$ for different values of Hartmann number $M_{0}$ and the couple stress parameter $l^{*}$. It is observed that, $P_{s}^{*}$ increases for increasing values of Hartmann number $M_{0}$ and couplestress parameter $l^{*}$. The 
variation of non-dimensional maximum steady film pressure $P_{S}^{*}(M)$ with non-dimensional minimum film height $h_{m}{ }^{*}$ for different values of $M_{0}$ and $l^{*}$ is shown in Fig. 3. It is observed that the non-dimensional maximum steady film pressure decreases with increasing values of non-dimensional minimum film height, further the non-dimensional maximum steady film pressure

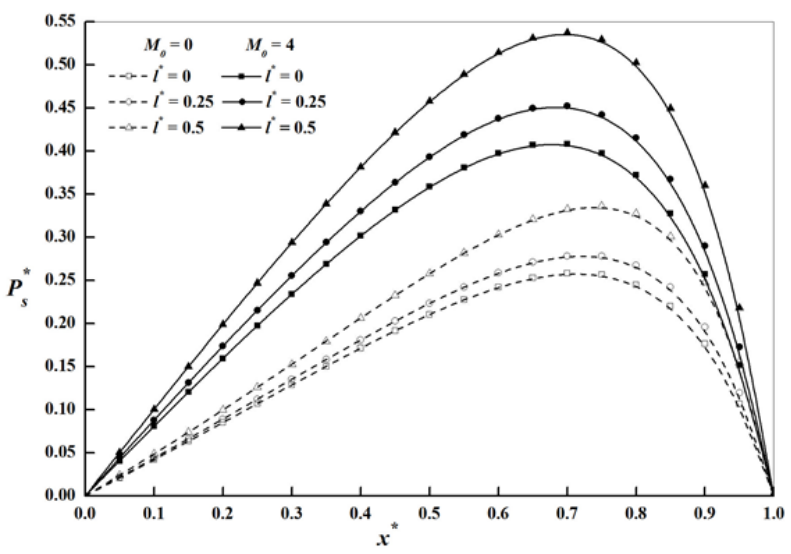

Fig. 2 Variation of steady film pressure $P_{s}^{*}$ with $x^{*}$ for different values of $M_{0}$ and $l^{*}$ with $h_{m}{ }^{*}$ $=1, \delta=1.5$

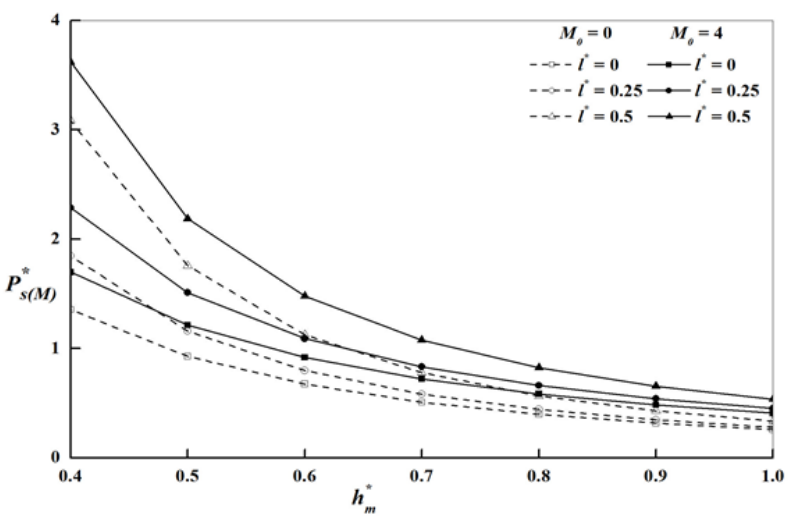

Fig. 3 Variation of steady maximum film pressure $P_{s}{ }^{*}(M)$ with $h_{m}{ }^{*}$ for different values of $M_{0}$ and $l^{*}$ with $\delta=1.5$

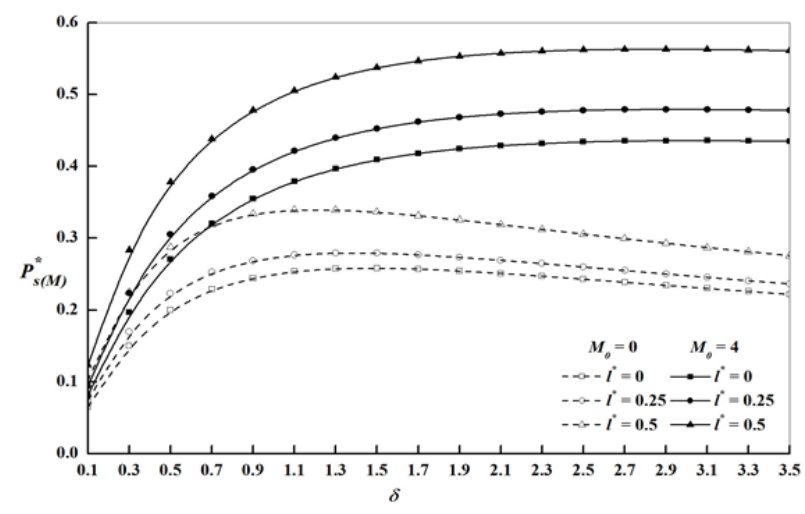

Fig. 4 Variation of steady maximum film pressure $P_{s}^{*}(M)$ with $\delta$ for different values of $M_{0}$ and $l^{*}$ with $h_{m}{ }^{*}=1$ increases with increasing values of Hartmann number $M_{0}$ and couplestress parameter $l^{*}$. Figure 4 shows the variation of steady maximum film pressure $P_{S}^{*}(M)$ with $\delta$ for different values of $M_{0}$ and $l^{*}$. It is observed that the steady maximum film pressure $P_{s}{ }^{*}(M)$ increases with increasing values of $\delta$ until maximum is obtained, there after decreases with increasing values of $\delta$.

\subsection{Steady load carrying capacity}

The variation of non-dimensional steady load carrying capacity $W_{s}^{*}$ with $\delta$ for different values of $M_{0}$ and $l^{*}$ is shown in Fig. 5. It is observed that the non-dimensional steady load carrying capacity $W_{s}^{*}$ increases with increasing values of $\delta$ until maximum is obtained, there after decreases with increasing values of $\delta$. Figure 6 shows the variation of non-dimensional steady load carrying capacity $W_{s}^{*}$ with non-dimensional minimum film height $h_{m}{ }^{*}$ for different values of $M_{0}$ and $l^{*}$. It is observed that the non-dimensional steady load carrying capacity $W_{s}^{*}$ decreases with increasing values of $h_{m}{ }^{*}$.

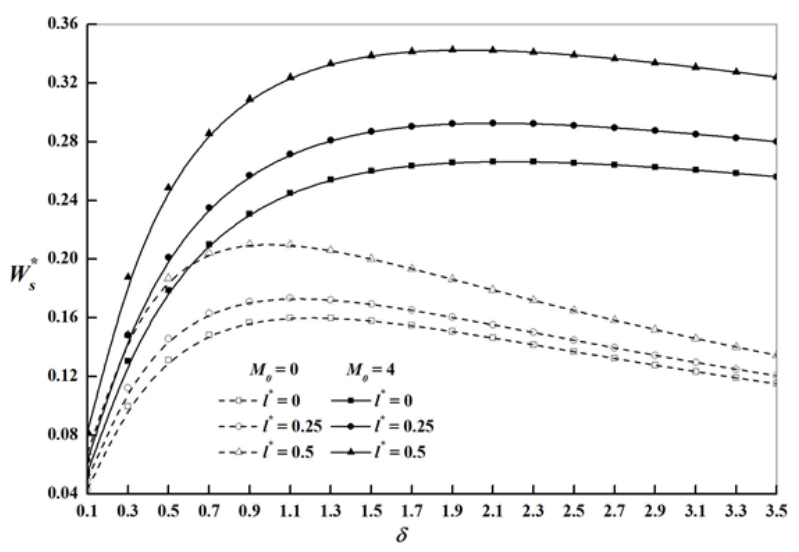

Fig. 5 Variation of steady load carrying capacity $W_{s}^{*}$ with $\delta$ for different values of $M_{0}$ and $l^{*}$ with $h_{m}{ }^{*}=1$

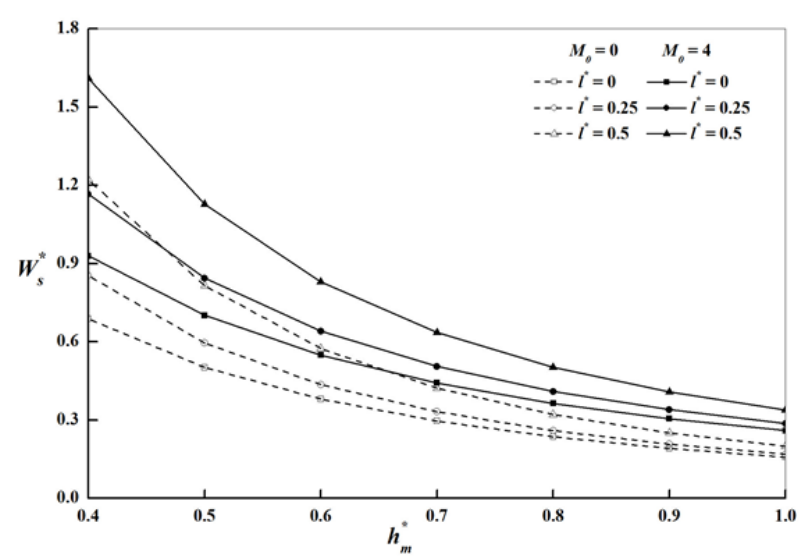

Fig. 6 Variation of steady load carrying capacity $W_{s}{ }^{*}$ with $h_{m}{ }^{*}$ for different values of $M_{0}$ and $l^{*}$ with $\delta=1.5$ 


\subsection{Dynamic stiffness}

Figure 7 depicts the variation of Variation of dynamic stiffness $K_{d}^{*}$ with $\delta$ for different values of $M_{0}$ and $l^{*}$. It is observed that the dynamic stiffness increases with increasing values of $\delta$ until maximum is obtained, there after decreases with increasing values of $\delta$. Figure 8 shows the variation of dynamic stiffness $K_{d}^{*}$ with non-dimensional minimum film height $h_{m}{ }^{*}$ for different values of $M_{0}$ and $l^{*}$. It is observed that the dynamic stiffness decreases with increasing values of $h_{m}{ }^{*}$,

\subsection{Damping coefficient}

Figure 9 depicts the variation of Variation of damping coefficient $D_{d}{ }^{*}$ with $\delta$ for different values of $M_{0}$ and $l^{*}$. It is observed that the damping coefficient decreases with increasing values of $\delta$. Figure 10 shows the variation of damping coefficient $D_{d}{ }^{*}$ with non-dimensional minimum film height $h_{m}{ }^{*}$ for different values of $M_{0}$ and $l^{*}$. It is observed that the damping coefficient decreases with increasing values of $h_{m}{ }^{*}$.

\section{Conclusions}

The effect MHD on couple stress fluid lubricated inclined slider bearing is presented. The modified

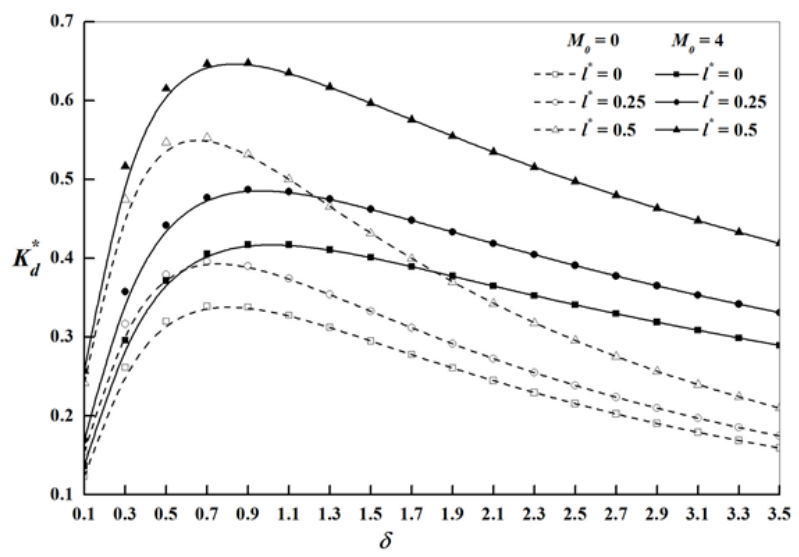

Fig. 7 Variation of dynamic stiffness $K_{d}{ }^{*}$ with $\delta$ for different values of $M_{0}$ and $l^{*}$ with $h_{m}{ }^{*}=1$

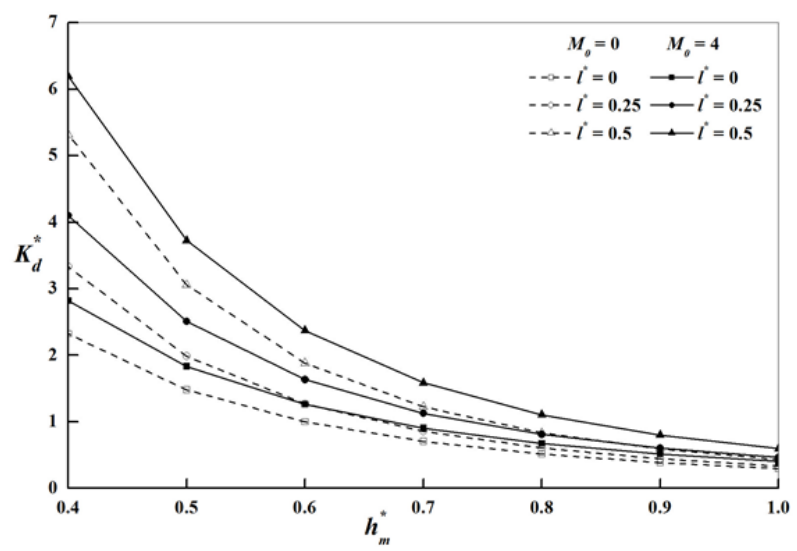

Fig. 8 Variation of dynamic stiffness $K_{d}{ }^{*}$ with $h_{m}{ }^{*}$ for different values of $M_{0}$ and $l^{*}$ with $\delta=1.5$
Reynolds equation is derived using Stokes micro-continuum theory and the magneto-hydrodynamic thin film lubrication theory. From the results obtained the following conclusions can be drawn.

1. For the plane slider bearing, the non-dimensional steady film pressure, non-dimensional steady load carrying capacity, Dynamic stiffness and Damping coefficient increases with the increasing values of the Hartmann number $M_{0}$ and the couple stress parameter $l^{*}$ as compared to the non magnetic case $\left(M_{0}=0\right)$ and the Newtonian case $\left(l^{*}\right.$ $=0)$.

2. For the plane slider bearing, the non-dimensional maximum steady film pressure, non-dimensional steady load carrying capacity and Dynamic stiffness increases with increasing values of $\delta$ until maximum is obtained, there after decreases with increasing values of $\delta$.

3. For the plane slider bearing, Damping coefficient decreases with increasing values of $\delta$.

4. For the plane slider bearing, the non-dimensional steady film pressure, non-dimensional steady load carrying capacity, Dynamic stiffness and

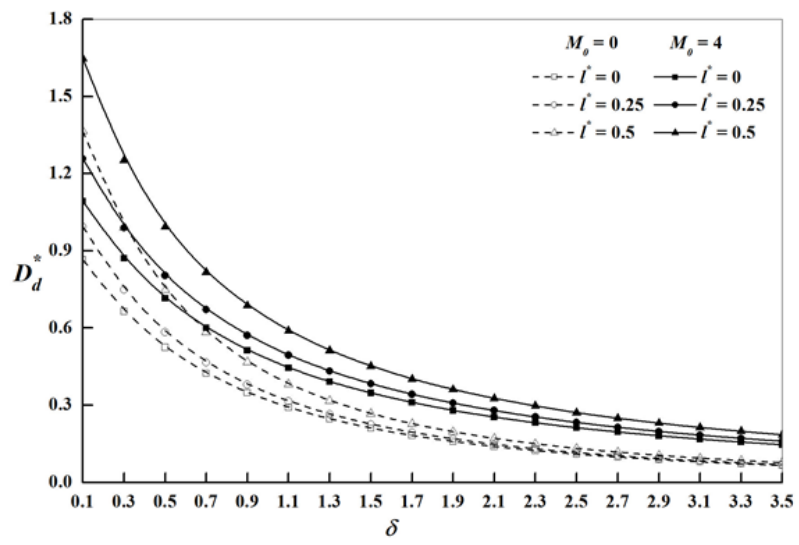

Fig. 9 Variation of damping coefficient $D_{d}{ }^{*}$ with $\delta$ for different values of $M_{0}$ and $l^{*}$ with $h_{m}{ }^{*}=1$

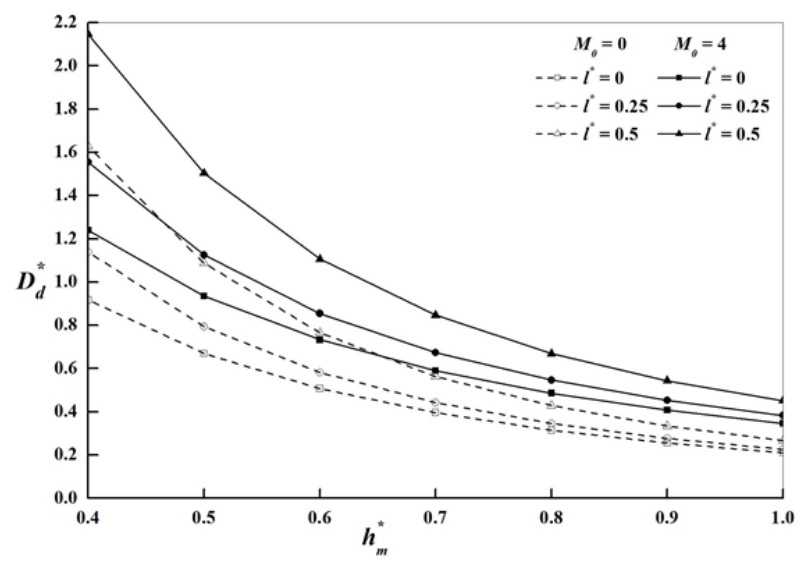

Fig. 10 Variation of damping coefficient $D_{d}{ }^{*}$ with $h_{m}{ }^{*}$ for different values of $M_{0}$ and $l^{*}$ with $\delta$ $=1.5$ 
Damping coefficient decreases with the increasing values of non-dimensional minimum film height $h_{m}{ }^{*}$.

\section{Nomenclature}

$B \quad$ width of the bearing

$B_{0} \quad$ applied magnetic field

$d \quad$ inlet-outlet thickness difference $\left(h_{1}-h_{m}\right)$

$D_{d}{ }^{*} \quad$ damping coefficient

$F \quad$ frictional force

$F^{*} \quad$ non-dimensional

frictional

$\left(=-F h_{m 0} / \mu U L\right)$

$F_{s}^{*} \quad$ non-dimensional steady frictional force

$h \quad$ film thickness

$h_{1} \quad$ inlet film thickness

$h_{1}{ }^{*} \quad$ non-dimensional inlet film thickness

$h_{m} \quad$ outlet film thickness

$h^{*} \quad$ non-dimensional film thickness $\left(=h / h_{m 0}\right)$

$K_{d}^{*} \quad$ dynamic stiffness

$l \quad$ couple stress parameter $(\eta / \mu)^{1 / 2}$ $l^{*} \quad$ non-dimensional couple stress parameter $\left(2 l / h_{m 0}\right)$

$L \quad$ Bearing length

$M_{0} \quad$ Hartmann number $\left(=B_{0} h_{m 0}(\sigma / \mu)^{1 / 2}\right)$

$p \quad$ pressure in the film region

$P^{*} \quad$ non-dimensional pressure $\left(=p h_{m 0}^{2} / \mu U L\right)$

$P_{s}^{*} \quad$ dimensionless steady film pressure

$x, y \quad$ rectangular coordinates

non-dimensional rectangular coordinates $\left(x^{*}=x / L\right)$

$u, v \quad$ velocity components in film region

$w \quad$ load carrying capacity

$W^{*}$ non-dimensional load carrying capacity $\left(=-w h_{m 0}^{2} / \mu U L^{2}\right)$

$W_{s}^{*} \quad$ dimensionless steady load carrying capacity

$\delta$ non-dimensional inlet-outlet thickness difference $\left(d / h_{m 0}\right)$ material constant characterizing couple stress viscosity coefficient electrical conductivity

\section{Appendix A}

$$
\begin{aligned}
& g_{11}=\frac{B^{2}}{\left(A^{2}-B^{2}\right)}\left\{\frac{\sinh (A h / l)-\sinh (A y / l)-\sinh A(h-y) / l}{\sinh (A h / l)}\right\} \\
& g_{12}=\frac{A^{2}}{\left(A^{2}-B^{2}\right)}\left\{\frac{\sinh (B h / l)-\sinh (B y / l)-\sinh B(h-y) / l}{\sinh (B h / l)}\right\} \\
& g_{13}=\frac{B^{2}\{\sinh (A h / l)-\sinh (A y / l)+\sinh A(h-y) / l\}}{\sinh (A h / l)\left\{\left(B^{2} / A\right) \tanh (A h / 2 l)-\left(A^{2} / B\right) \tanh (B h / 2 l)\right\}} \\
& g_{14}=\frac{A^{2}\{\sinh (B h / l)-\sinh (B y / l)+\sinh B(h-y) / l\}}{\sinh (B h / l)\left\{\left(B^{2} / A\right) \tanh (A h / 2 l)-\left(A^{2} / B\right) \tanh (B h / 2 l)\right\}} \\
& A=\left[\frac{1+\left\{1-\left(4 l^{2} M_{0}^{2} / h_{m 0}^{2}\right)\right\}^{1 / 2}}{2}\right]^{1 / 2} \\
& B=\left[\frac{1-\left\{1-\left(4 l^{2} M_{0}^{2} / h_{m 0}^{2}\right)\right\}^{1 / 2}}{2}\right]^{1 / 2} \\
& g_{21}=\frac{\sinh \{(y-h) / \sqrt{2} l\}+\sinh (y / \sqrt{2} l)-\sinh (h / \sqrt{2} l)}{\sinh (h / \sqrt{2} l)} \\
& g_{22}=\frac{y \cosh \{(y-h) / \sqrt{2} l\}+y \cosh (y / \sqrt{2} l)-h \operatorname{coth}(h / 2 \sqrt{2} l) \sinh (h / \sqrt{2} l)}{2 \sqrt{2} l \sinh (h / \sqrt{2} l)} \\
& g_{23}=\frac{y \sinh \{(y-h) / \sqrt{2} l\}+y \sinh (y / \sqrt{2} l)-h \sinh (y / \sqrt{2} l)}{6 l \sinh (h / \sqrt{2} l)-\sqrt{2} h}
\end{aligned}
$$




$$
\begin{aligned}
& g_{24}=\frac{2 \cosh \{(y-h) / \sqrt{2} l\}+2 \cosh (y / \sqrt{2} l)-2 \cosh (h / \sqrt{2} l)-2}{3 \sqrt{2} \sinh (h / \sqrt{2} l)-(h / l)} \\
& g_{31}=\frac{\cosh A_{1} y \cos B_{1}(y-h)-\cos B_{1} y \cosh A_{1}(y-h)}{\left(\cosh A_{1} h-\cos B_{1} h\right)} \\
& g_{32}=\frac{\cot \theta\left\{\sinh A_{1} y \sin B_{1}(y-h)-\sin B_{1} y \sinh A_{1}(y-h)\right\}+\left(\cosh A_{1} h-\cos B_{1} h\right)}{\left(\cosh A_{1} h-\cos B_{1} h\right)} \\
& g_{33}=\frac{\cot \theta\left\{\sin B_{1} y \sinh A_{1}(y-h)+\sinh A_{1} y \sin B_{1}(y-h)\right\}+\left(\cos B_{1} h+\cosh A_{1} h\right)}{\left(B_{1}-A_{1} \cot \theta\right) \sin B_{1} h+\left(A_{1}+B_{1} \cot \theta\right) \sinh A_{1} h} \\
& g_{34}=\frac{\cos B_{1} y \cosh A_{1}(y-h)+\cosh A_{1} y \cos B_{1}(y-h)}{\left(B_{1}-A_{1} \cot \theta\right) \sin B_{1} h+\left(A_{1}+B_{1} \cot \theta\right) \sinh A_{1} h} \\
& A_{1}=\sqrt{M_{0} / l h_{m 0}} \cos (\theta / 2) \\
& B_{1}=\sqrt{M_{0} / l h_{m 0}} \sin (\theta / 2) \\
& \theta=\tan ^{-1}\left(\sqrt{4 l^{2} M_{0}^{2} / h_{m 0}^{2}-1}\right) \\
& \xi_{11}=\frac{\left(A^{* 2} / B^{*}\right) \tanh \left(B^{*} h^{*} / l^{*}\right)-\left(B^{* 2} / A^{*}\right) \tanh \left(A^{*} h^{*} / l^{*}\right)}{A^{* 2}-B^{* 2}} \\
& \xi_{12}=\frac{\left(A^{* 2} / l^{*}\right) \operatorname{sech}^{2}\left(B^{*} h^{*} / l^{*}\right)-\left(B^{* 2} / l^{*}\right) \operatorname{sech}^{2}\left(A^{*} h^{*} / l^{*}\right)}{A^{* 2}-B^{* 2}} \\
& \xi_{21}=(3 / \sqrt{2}) \tanh \left(h^{*} / \sqrt{2} l^{*}\right)-\left(h^{*} / 2 l^{*}\right) \operatorname{sech}^{2}\left(h^{*} / \sqrt{2} l^{*}\right) \\
& \xi_{22}=\operatorname{sech}^{2}\left(h^{*} / \sqrt{2} l^{*}\right)\left\{\left(1 / l^{*}\right)+\left(h^{*} / \sqrt{2} l^{* 2}\right) \tanh \left(h^{*} / \sqrt{2} l^{*}\right)\right\} \\
& \xi_{31}=\frac{\left(B^{*}-A^{*} \cot \theta^{*}\right) \sin B^{*} h^{*}+\left(A^{*}+B^{*} \cot \theta^{*}\right) \sinh A^{*} h^{*}}{M_{0}\left(\cos B^{*} h^{*}+\cosh A^{*} h^{*}\right)} \\
& \xi_{32}=\frac{2}{l^{*}}\left\{\frac{1+\cot \theta^{*} \sin B^{*} h^{*} \sinh A^{*} h^{*}+\cos B^{*} h^{*} \cosh A^{*} h^{*}}{\left(\cos B^{*} h^{*}+\cosh A^{*} h^{*}\right)^{2}}\right\}
\end{aligned}
$$

\section{References}

[1] Agrawal, V. K., "Inertia Effects in Hydromagnetic Inclined Slider Bearing," Japanese Journal of Applied Physics, 9, 7, 1970, 820-824.

[2] Kuzma, D. C., "The Magnetohydrodynamic Journal Bearing," Journal of Basic Engineering, 85, 3, 1963, 424-427.

[3] Naduvinamani, N. B., Fathima, S. T. and Hanumagowda, B. N., "Magneto-Hydrodynamic Couplestress Squeeze Film Lubrication of Circular Stepped Plates," Proceedings of the Institution of Mechanical Engineers, Part J: Journal of Engineering Tribology, 225, 3, 2011, 111-119.

[4] Lin, J. R., "Dynamic Characteristics for Wide Magneto-Hydrodynamic Slider Bearings with a Power-Law Film Profile," Applied Mathematical Modelling, 36, 9, 2012, 4521-4528.

[5] Fathima, S. T., Naduvinamani, N. B. and Marulappa, S. H., "Hydromagnetic Squeeze Films between Porous Rectangular Plates with
Couplestress Fluids," Tribology Online, 7, 4, 2012, 258-266.

[6] Oliver, D. R., "Load Enhancement Effects due to Polymer Thickening in a Short Model Journal Bearing," Journal of Non-Newtonian Fluid Mechanics, 30, 2-3, 1988, 185-196.

[7] Scott, W. and Suntiwattana, P., "Effect of Oil Additives on the Performance of a Wet Friction Clutch Material," Wear, 181-183, 2, 1995 , 850-855.

[8] Spikes, H. A., "The Behaviour of Lubricants in Contacts; Current Understanding and Future Possibilities," Proceedings of the Institution of Mechanical Engineers, Part J: Journal of Engineering Tribology, 208, 1, 1994, 3-15.

[9] Ariman, T., Turk, M. A. and Sylvester, N. D., "Microcontinuum Fluid Mechanics-A Review," International Journal Engineering Science, 11, 8, 1973, 905-930.

[10] Ariman, T., Turk, M. A. and Sylvester, N. D., "Applications of Microcontinuum Fluid 
Mechanics," International Journal Engineering Science, 12, 4, 1974, 273-293.

[11] Stokes, V. K., "Couple Stresses in Fluids," The Physics of Fluids, 9, 9, 1966, 1709-1715.

[12] Kashinath, B., "Squeeze Film Lubrication between Parallel Stepped Plates with Couplestress Fluids," International Journal of Statistika and Mathematica, 3, 2, 2012, 65-69.

[13] Lin, J. R., Lu, R. F., Liao, W. H. and Kuo, C. C., "Effect of Couple Stresses and Convective Inertia Forces in Parallel Circular Squeeze-Film Plates," Industrial Lubrication and Tribology, 56, 6, 2004, 318-323.

[14] Naduvinamani, N. B. and Marali, G. B., "Dynamic Reynolds Equation for Micropolar Fluid Lubrication of Porous Slider Bearings," Journal of Marine Science and Technology, 16, 3, 2008, 182-190.
[15] Bujurke, N. M., Patil, H. P. and Bhavi, S. G., "Porous Slider Bearing with Couple Stress Fluid," Acta Mechanica, 85, 1, 1990, 99-113.

[16] Gupta, R. S. and Sharma, L. G., "Analysis of Couple Stress Lubricant in Hydrostatic Thrust Bearing," Wear, 125, 3, 1988, 257-269.

[17] Naduvinamani, N. B., Fathima, S. T. and Hiremath, P. S., "Effect of Surface Roughness on Characteristics of Couplestress Squeeze Film between Anisotropic Porous Rectangular Plates," Fluid Dynamics Research, 32, 5, 2003, 217-231.

[18] Lin, J. R., Hung, C. R., Hsu, C. H. and Lai, C, "Dynamic Stiffness and Damping Characteristics of One-Dimensional Magneto-Hydrodynamic Inclined-Plane Slider Bearings," Proceedings of the Institution of Mechanical Engineers, Part J: Journal of Engineering Tribology, 223, 2, 2009, 211-219. 